\title{
A Web-Based Institutional DICOM Distribution System with the Integration of the Clinical Trial Processor (CTP)
}

\author{
K. Y. E. Aryanto • A. Broekema • R. G. A. Langenhuysen • \\ M. Oudkerk • P. M. A. van Ooijen
}

Received: 17 July 2014 / Accepted: 29 December 2014 / Published online: 3 March 2015

(C) The Author(s) 2015. This article is published with open access at Springerlink.com

\begin{abstract}
To develop and test a fast and easy rule-based webenvironment with optional de-identification of imaging data to facilitate data distribution within a hospital environment. A web interface was built using Hypertext Preprocessor (PHP), an open source scripting language for web development, and Java with SQL Server to handle the database. The system allows for the selection of patient data and for de-identifying these when necessary. Using the services provided by the RSNA Clinical Trial Processor (CTP), the selected images were pushed to the appropriate services using a protocol based on the module created for the associated task. Five pipelines, each performing a different task, were set up in the server. In a 75 month period, more than 2,000,000 images are transferred and de-identified in a proper manner while 20,000,000 images are moved from one node to another without de-identification. While maintaining a high level of security and stability, the proposed system is easy to setup, it integrate well with our clinical and research practice and it provides a fast and accurate vendor-neutral process of transferring, de-identifying, and storing DICOM images. Its ability to run different deidentification processes in parallel pipelines is a major advantage in both clinical and research setting.
\end{abstract}

This article is part of the Topical Collection on Patient Facing Systems.

K. Y. E. Aryanto $(\bowtie) \cdot$ M. Oudkerk $\cdot$ P. M. A. van Ooijen Department of Radiology, Center for Medical Imaging - North East Netherlands (CMINEN), University of Groningen, University Medical Center Groningen, Hanzeplein 1, Postbus 30001, 9700, RB Groningen, The Netherlands

e-mail: k.y.e.aryanto@umcg.nl

A. Broekema

Department of Radiology, University Medical Center Groningen, Groningen, The Netherlands

R. G. A. Langenhuysen

Rogan B.V., Huis ter Heide, Utrecht, The Netherlands
Keywords DICOM distribution system - Web based system · Clinical trial processor $\cdot$ Vendor-neutral system

\section{Introduction}

Over the last decade, Picture Archiving and Communication Systems (PACS) evolved from a predominantly radiological service into the fundament of the image management system utilized throughout the entire healthcare enterprise for clinical practice and research [1-4]. It integrates multimedia, information, and communication technologies, providing practically unlimited amounts of data for patient care, research and teaching [5].

PACS integration with other information systems has made medical image distribution to play an important role in the entire clinical process including the interpretation, processing and analysis of imaging data [6,7]. Distribution of images and information to clinicians in a timely manner is therefore required to facilitate clinical work-up. Although current highspeed networks allow exchange or transfer of data from one node to another at acceptable speed, optimization of the processing of digital images during the distribution is also a prerequisite to meet the clinical and research needs.

The Digital Imaging and Communication in Medicine (DICOM) standard has been widely adopted for recording and sharing of digital medical images [8]. A DICOM image consists of a header with patient and exam data firmly connected with the image pixel data in a single file. The DICOM header contains a large amount of information related to the pixel data it belongs to, including identifiable information about the patient, the study, and the institution. Because of this inclusion of possible sensitive data, de-identification of 
DICOM images is often required in data exchange for clinical research $[9,10]$.

The information embedded in the DICOM data should be handled carefully to ensure the security of patients' identity, especially when transfers are intended for a clinical trial or research. The importance of security issue had motivated research in the utilisation of health information within data exchange and the use of information system technology [11-13]. However, the process of de-identifying the data should be implemented such that it provides ease of use and simple configuration in order to facilitate all users - including inexperienced ones - to adequately fulfil the task of ensuring the patient data protection. The imaging IT infrastructure within an institution may consists of many smaller systems that support each other Of these systems, some are often not integrated within the normal workflow but operated as standalone applications thus reducing the ease of use and increasing the risk of errors and mistakes. Therefore, other than just the easeof-use of the systems introduced to handle a certain task, the ease of integration of those systems have also become an important issue to adequately fulfill the requirements of smooth integration into the daily work processes. Therefore, besides providing an easy to use process, health institutions are also required to develop systems that are ready to be integrated into the normal workflow using current standards.

To facilitate data distribution within a hospital environment, a web-based system called RadTransceiver was built. The system was implemented as a fast and easy web-environment, providing an integrated rule-based distribution and optional de-identification of imaging data.

\section{Materials and methods}

The server side of the RadTransceiver runs under the Microsoft Windows Server 2003 operating system. All RadTranceiver services were developed using Java [14], a multi platform general purpose object oriented programming language, with Microsoft SQL Server [15] to handle the database management system. The Graphical User Interface (GUI) was designed using PHP: Hypertext Preprocessor (PHP) [16], a general-purpose scripting language which is especially suitable for Web development and which can be embedded into HyperText Markup Language (HTML). Web-based system is deployed to enable the broader range of utilisation, the ease of use and understand, with the interfaces that suit the variations in computer literacy [17].

User rights in accessing RadTransceiver are divided into three types: superuser, managerial user and ordinary user. A superuser has unrestricted access to all features of the system. This category of users contains the administrators who are responsible to manage and supervise the whole system and take actions over unwanted activities or erroneous usage of the system. Managerial users have access to the managerial features of the system to view all image processes and their status. Ordinary users have limited rights, can only view and access their own initiated processes and have no access to the critical features of the system or information which belongs to other users. Superusers and managerial users have the ability to create and make changes of the transfer rules while ordinary users are only able to perform image transfer based on the transfer rules that are related to their login credentials.

There are two kinds of image transfer that could be initiated by the users. First is a direct transfer from source to destination node, for example the transfer to a viewer in the Electronic Patient Record (EPR) system. With the second type of transfer the images have to pass through a processor before the images are transferred to the destination node (Fig. 1).

To perform the processing of the images the services provided by the Clinical Trial Processor (CTP) are utilized [18]. CTP is a stand-alone DICOM Application Entity (AE) developed by the Radiological Society of North America (RSNA) [19]. It has the capability of using multiple, independently configured, pipelines for running several processes in parallel. With this flexibility and configurability of pipelines the deidentification of selected patient data can be performed using a variety of pertinent rules and regulations [20]. In our DICOM distribution system, each pipeline runs its own CTP DICOM import service. This import service consists of a Storage Service Class Provider (Storage SCP), accepting images from other DICOM nodes (e.g., PACS or modality) and putting them in a queue for further process.

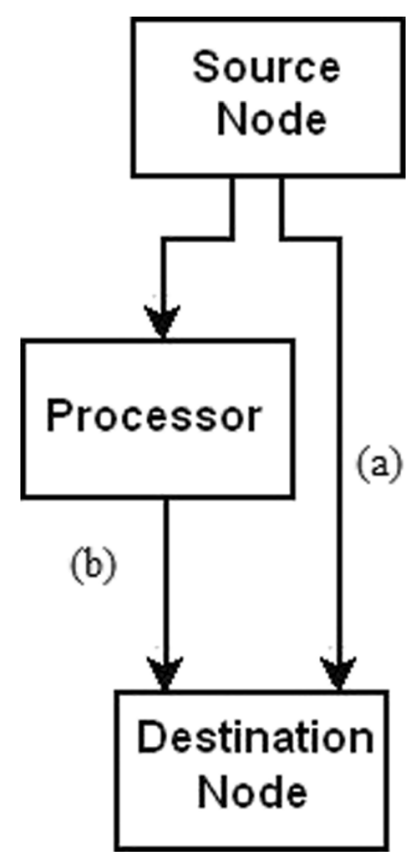

Fig. 1 Two kinds of image transfer: a a direct transfers from one node to the destination node (such as from PACS to viewers); $\mathbf{b}$ a transfer through a processor before images reach the destination 
In our setup, three possible pipelines are defined (Fig. 2).

1. A pipeline without de-identification sending data into a file system.

2. A pipeline with de-identification that exports the images in a specific directory or network attached storage.

3. A pipeline with de-identification sending the DICOM images to a DICOM node using the DICOM export service.

In case of transfer without de-identification, the pipeline consists of a Storage SCP as part of the Import Service followed by a Storage Service Class User (Storage SCU) as part of the Export Service. Note that a Service Class Provider (SCP) is a DICOM node or device that provides specific DICOM network services such as for instance those offered by the Storage Class defined by DICOM. A Service Class User (SCU) is the client role which makes use of DICOM Services offered by an SCP. The RadTransceiver is an Application Entity (AE) that is an SCU as well as an SCP for the DICOM Storage Service Class. An AE is a node or an application providing DICOM Services within the network. In RadTransceiver the storage service class mechanism saves images into specific folders defined in the configuration file.

When required, a pipeline with an anonymizer is configured to de-identify images before they are stored in a DICOM node. The execution of a de-identification task will trigger a service to add a record for each image to be de-identified into a confidential database, registering the identity mapping between original and de-identification ID.

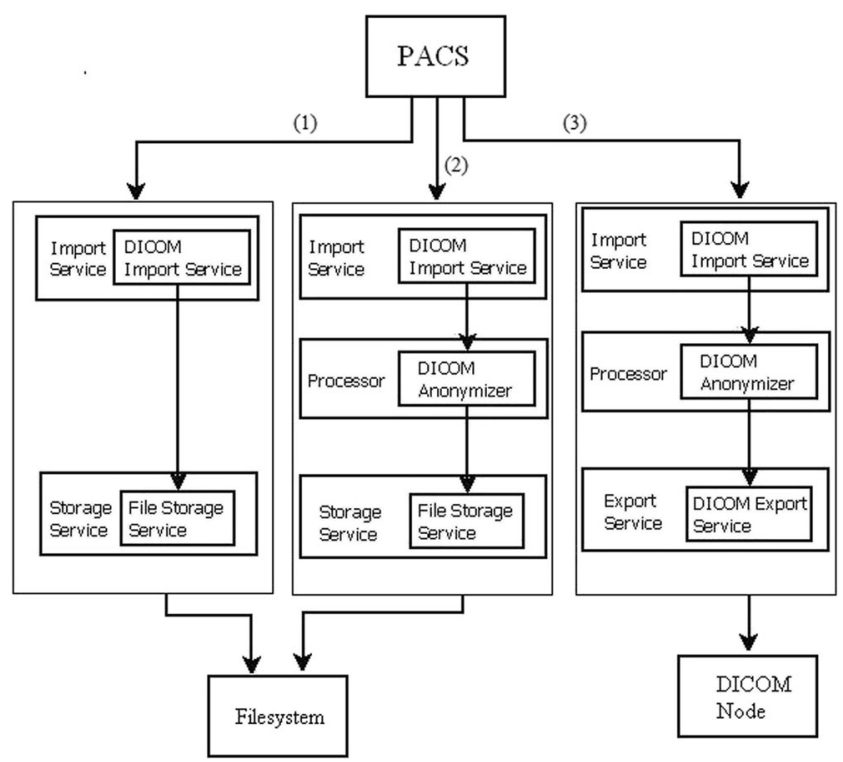

Fig. 2 Data are transferred using one from three types of CTP pipelines set for the processor: 1) a pipeline without de-identification scheme to a local file system; 2) a pipeline with a de-identification process before the images are stored in a specific directory; 3) a pipeline with a deidentification process before the images are sent to another DICOM node by the export service
A separate service monitors the amount of files being stored. When the amount of files in one task matches the amount as registered in the database, the service will mark the transfer task as a completed process.

\section{Results}

After being logged in into RadTransceiver, a user initiates and registers the transfer of DICOM data through the web interface by entering a patient ID and selecting the appropriate images to be transferred from the PACS. DICOM transfer of the selected images to another DICOM node without deidentification is performed instantly while all other transfers are pushed into a processor service. Using the processor, images can be transferred from the PACS or modality into the predefined storage destination or sent through a process of image de-identification using a specific profile before they are stored.

The managers of RadTransceiver can create one or more user-specific module(s) related to the type of clinical research study and associate a transfer protocol for that user/study combination. The transfer protocol destination node defines how it has to be stored (file system or DICOM) and whether it has to be de-identified or not. This allows to configuring and utilizing different modules for specific tasks and users. These

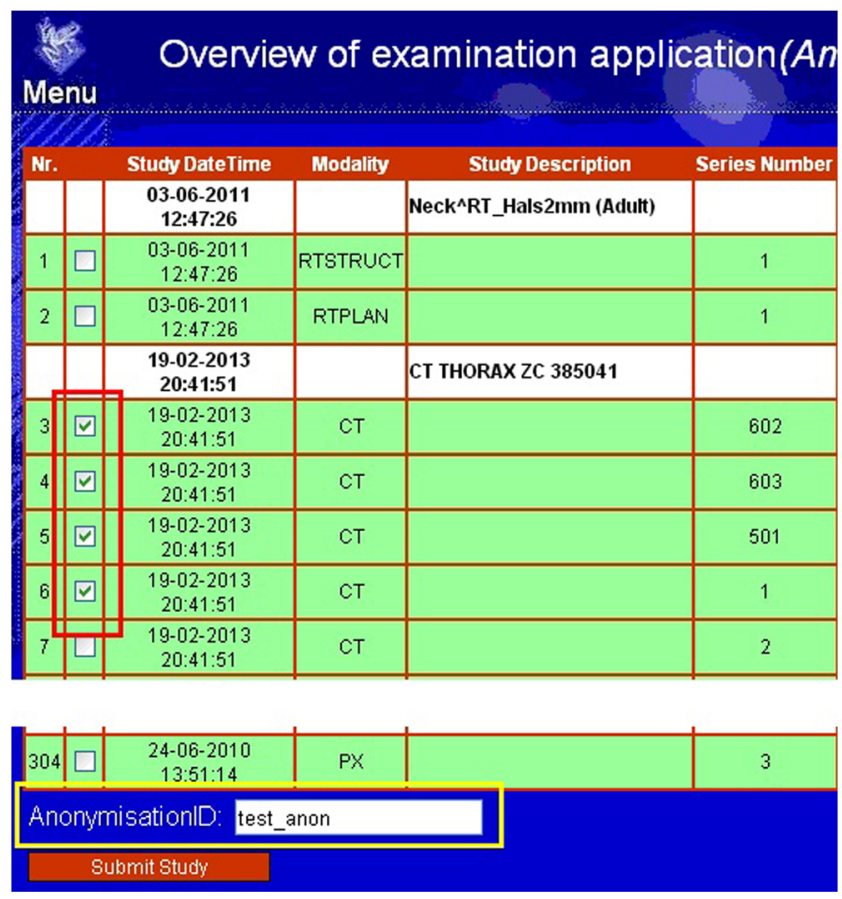

Fig. 3 Web interface. The web interface shows the results of a search for a patient from the PACS based on the patient ID, selection of images (surrounded by the red rim on the left side), and entry of an anonymizationID (surrounded by the yellow rim) in the bottom part of the web page 
Fig. 4 The web interface shows the selected images are queued and waiting to be processed. The status indicates that images are in the "waiting" list

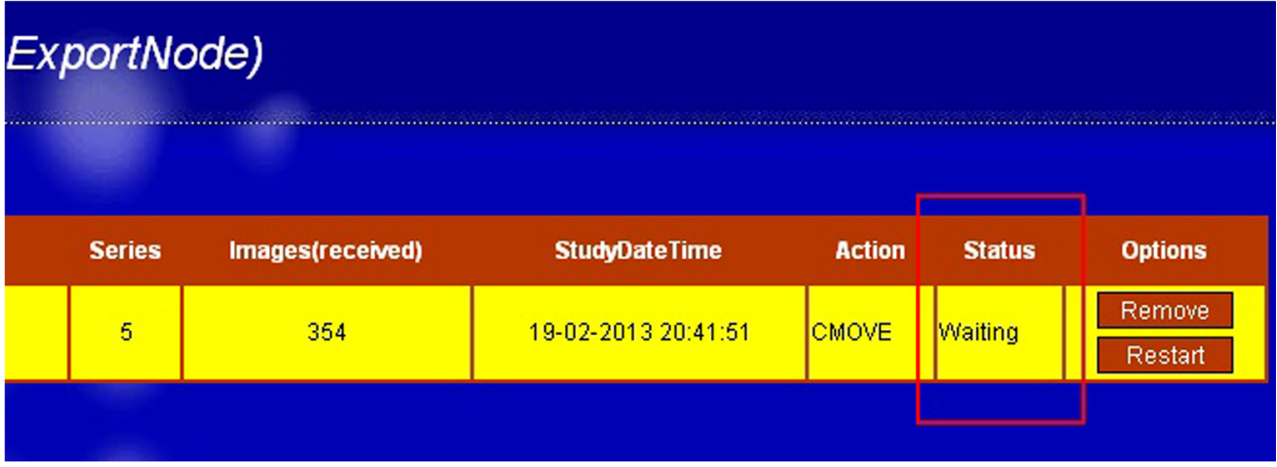

modules are linked to the user account and thus a user can only execute those modules assigned to his/her user profile.

The result of a patient ID based search from the PACS provides a list of selectable items of available study entities and serie within those entities in DICOM files. Date, time and study descriptions are shown for each study entity. The date and time, modality type, series number, series description, number of images, and accession number are shown for each series. The user then selects the required series by ticking the checkboxes at the beginning of the corresponding row. Further processing will be done after the submission button is clicked by the user. The de-identification ID is entered by the user using an input box provided at the bottom of the page. When no de-identification ID is provided a time-stamp based de-identification ID will be automatically generated. The search results, study entity selection and entry of identification-ID are shown in Fig. 3.

Some modules are triggered automatically from the EPR and designed to transfer data from PACS directly to the EPR without user interaction. Since EPR users can send requests of image retrieval through the system, from the image viewing process, the EPR itself can be considered as a user that can initiate image transfers from PACS.

Users can track the status of their own transfers by accessing the web interface for the associated process showing a list of jobs in 'waiting' (Fig. 4) and 'processing' (Fig. 5) state.

RadTransceiver also has a priority system that affects the order of processing of the images. Transfer processes with a higher priority always appear in the top rows of the queue and are thus handled right after the current active process has finished. When there is more than one process with the same priority they will be sorted based on their time of requests, following the 'first in - first out' principle.

The initiation of a de-identification task by the user will generate a mapping of the original, unique, identifier into a de-identification identifier both in the database and the lookup-table. The update on the database is required to register the transfer itself and to record the amount of images being sent to be matched in the last stage of the process. Meanwhile, the creation of id-mapping on the lookup-table will be used in the de-identification process itself. The lookup-table is updated continuously and only contains the id-mapping of active transfers. The mapping will be removed when a transfer task is finished.

Five pipelines composed of a minimum of one DICOM import service and either a DICOM storage or export service are defined in the CTP server to provide different DICOM nodes with images according to their specific needs. With the DICOM export service set in the configuration, DICOM images received by the CTP were stored at a different DICOM node within our institution. The resulting images were all stored at the correct DICOM node without any duplication occurring. The anonymizer was set only when the related transfer required de-identification. Figure 6 shows the entire system.

Processes can be monitored by observing the status of the process in the web pages. When failures occur, the user can restart or remove the tasks using the corresponding buttons on the rightmost sisde of the task-row. Figure 7 shows the finished process in the web interface.
Fig. 5 The web interface shows the selected images are being handled by the system. The status indicates that the images are being processed

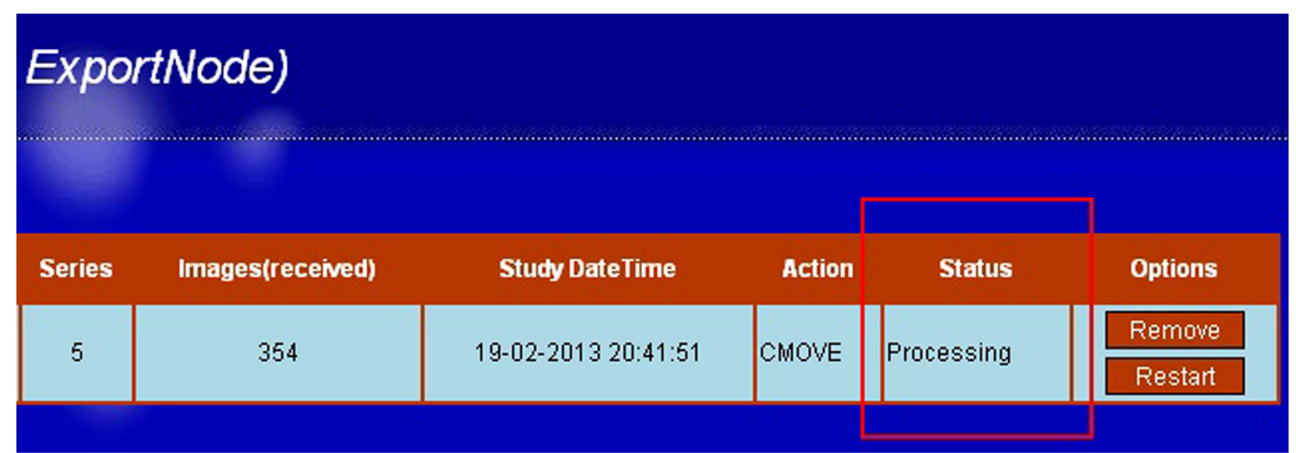


Fig. 6 Web based DICOM distribution system with CTP integrated as its image processor

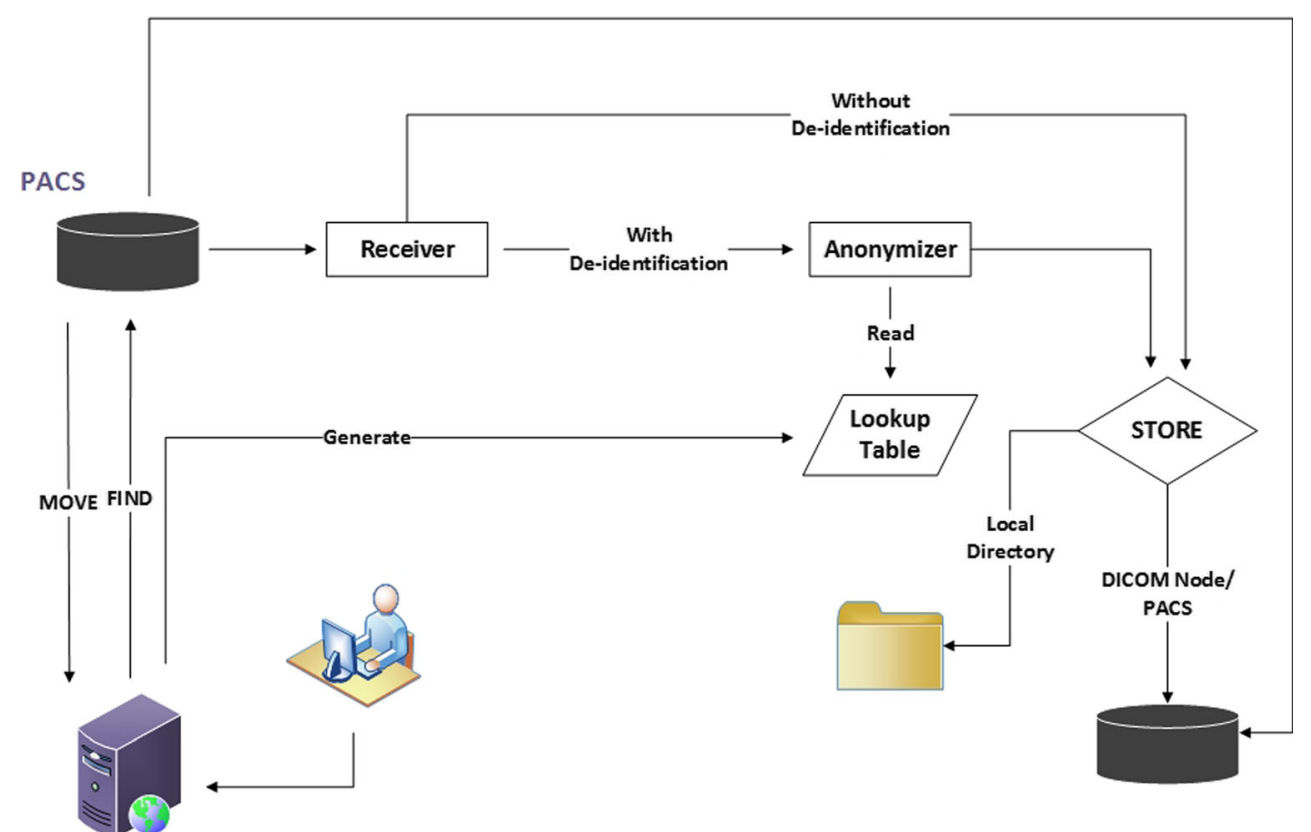

The system has transferred more than 24 millions images during 75 months of stable operation (October 2006 until December 2012). More than 2,000,000 images were transferred and de-identified in a proper manner while the rest $(20,000$, 000 ) were only moved from one node to another without deidentification. Of the data transferred, $10 \%$ was de-identified and thus used for clinical research trials or teaching, the remaining data was transferred for patient care. The data without de-identification was transferred within our institution to move older imaging data from one system (the PACS) to the other (institutional web browser). In Fig. 8 the total number of images which have been transferred within 75 months until December 2012 is shown and Fig. 9 displays the difference regarding images transferred with the identification profiles and without it. By performing multiple transfers using the system, time from request to delivery of the images was computed covering the whole process of transfer, de-identification, and data strore. The system delivers images at an average rate of four CT images per seconds (Table 1), and MR images at an average rate of eight images per seconds.

\section{Discussion}

A DICOM data distribution system was developed to provide a fast and easy environment to facilitate the distribution of medical images within an institution. The use of commonly known web based technologies and the flexible processor make it easy to implement and adapt to specific use.

DICOM de-identification covers the security of patientrelated data, but data trackback is still possible by retrieving the de-identification entry in the conversion table. Therefore, the access to the clinical research study information system database and conversion table is limited to authorized personnel and can be obtained through an internal network only.

The large number of images transferred without deidentification is caused by the fact that this setup is also used to facilitate the retrieval of image data into an EPR. The DICOM web-viewer integrated into our EPR is limited to about 1 year of storage. To enable easy access to older data, EPR users can automatically retrieve the historical data from their patient with one click within the EPR system. Requests through

Fig. 7 Finished task shown in the web interface. The status (surrounded by the yellow rim), is indicated by "completed". The buttons next to the status are used for restarting or removing the task

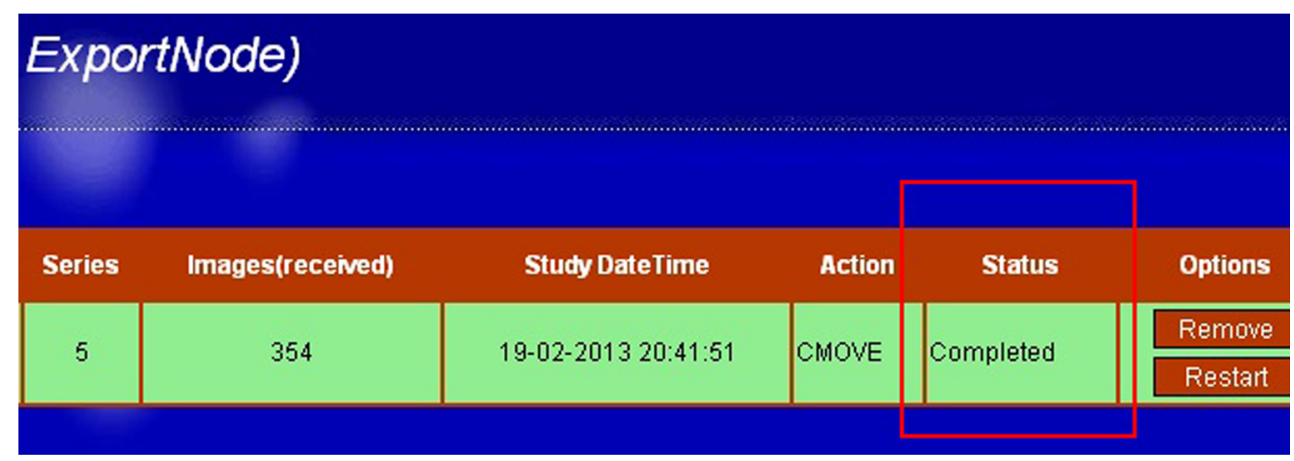


Fig. 8 Total number of images which have been transferred annually since October 2006 until December 2012

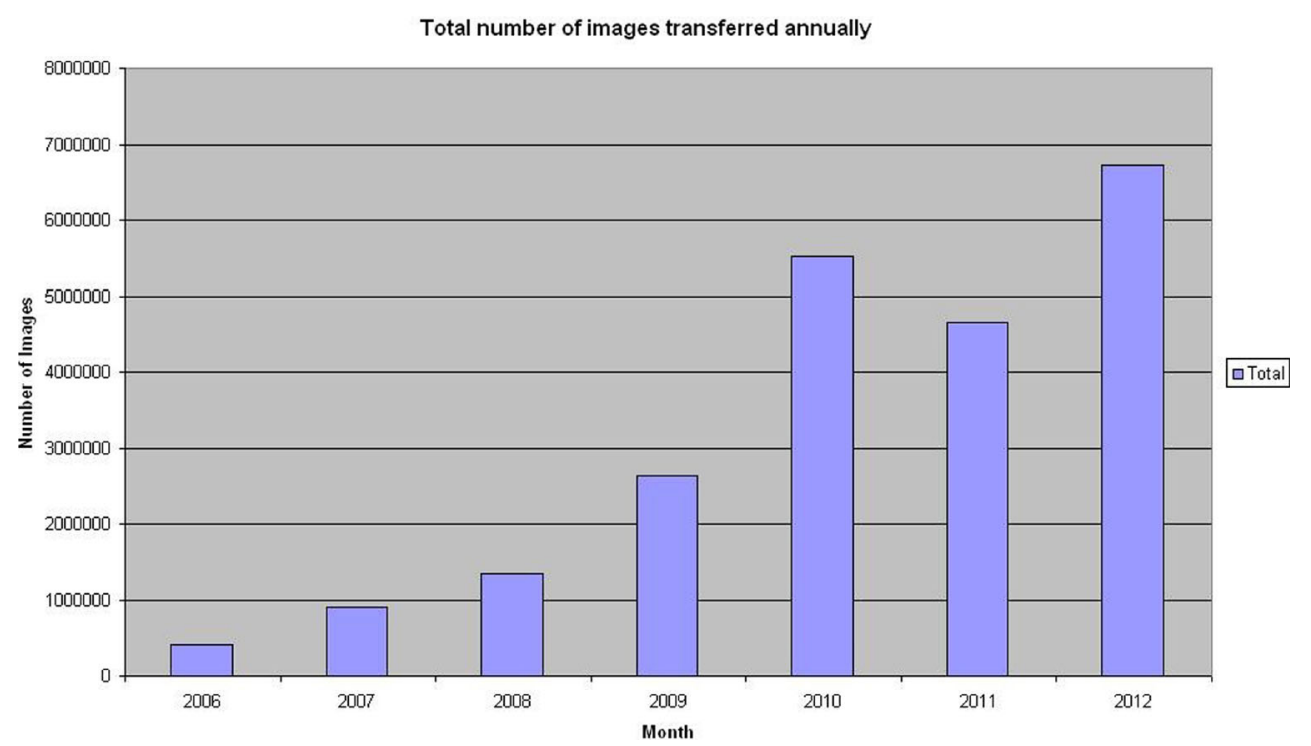

the EPR are considered as high-priority tasks and therefore, the data will immediately be transferred from PACS to the EPR.

In many institutions the production of de-identified data is performed from the PACS. Thus, it requires the use of a full PACS workstation for the researcher. This involves a costly PACS license and blocks the workstation for actual clinical reporting use for the time required for the de-identification. Alternatively, users get the non de-identified DICOM data locally and perform the de-identification process manually. This leads to the possibility of use of a wide variety of not properly managed tools and thus cannot ensure patient data and identity safety. Using RadTransceiver, the access is provided through a simple, password-protected, webpage which can be accessed from any PC in a hospital; the deidentification process is controlled and supervised centrally and is vendor neutral.
In some cases trace-back of the actual patient behind the de-identified data is required, for example in the case of incidental findings in scans made for a research study. Therefore, our de-identification method, sometimes referred to as pseudonymization, uses a lookup-table which allows tracing back the original patient information. However, only necessary data are pseudonymized while the remaining fields are made anonymous. This is the reason why CTP is used within our system; besides transferring and processing the DICOM data in proper manner to provide patient data security, integrity and confidentiality, it also provides simple yet flexible and extensive configuration of DICOM de-identification profiles.

The lookup-table consist of an ID-mapping between the original and the de-identified ID and was used by the system to record and guide the de-identification process. However, besides the replacement of the patient ID as used in the
Fig. 9 Number of images being transferred with and without deidentification profile

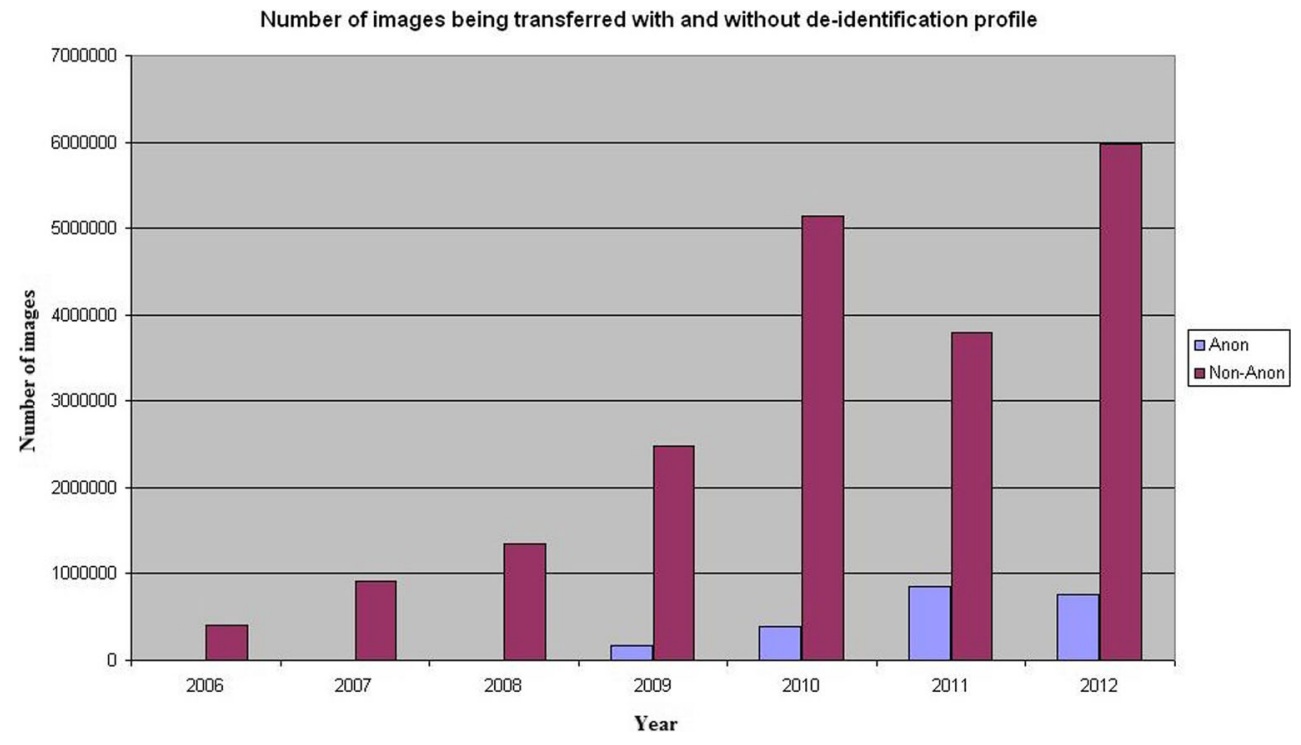


Table 1 Several transfers using CT and MR images, giving a rate of four CT images per second and eight MR images per second

\begin{tabular}{lcrll}
\hline Type & $\begin{array}{l}\text { Number of } \\
\text { images }\end{array}$ & $\begin{array}{l}\text { Size } \\
\text { (MB) }\end{array}$ & $\begin{array}{l}\text { Time } \\
\text { (Seconds) }\end{array}$ & $\begin{array}{l}\text { Images per } \\
\text { second }\end{array}$ \\
\hline CT & 100 & 52 & 22 & 5 \\
& 500 & 251 & 120 & 4 \\
& 1000 & 505 & 253 & 4 \\
& 2000 & 1000 & 460 & 4 \\
MR & 100 & 13 & 12 & 8 \\
& 1000 & 108 & 124 & 8 \\
& 5000 & 615 & 627 & 8 \\
\hline
\end{tabular}

lookup-table a whole set of 50 DICOM header tags are deidentified during the de-identification process (see Table 2). These 50 tags are considered to be the possible cause of data breach when they are exposed to a third party either by the element itself or combination with other elements. All 50 elements are replaced by the de-identification ID, a blank, or a more general representation. Although this provides a proper de-identification of the DICOM header, a more comprehensive de-identification, including blanking of part of the image, should be considered in accordance to the DICOM
Supplement 142 Clinical Trial De-identification profiles to ensure the full protection of patient's private data [21].

The export and store services provided by the processor can also be utilized for image sharing between health enterprises. De-identified images can be directly uploaded using a DICOM or Hypertext Transfer Protocol (HTTP) or stored into a specific directory of the system that will transfer images to outside the institution. Therefore, this system would be adaptable for the implementation of a large multi-centre clinical trial study. The interface of the DICOM distribution system was built using web technologies, therefore, wider access is possible. The de-identification profiles will be kept controlled since the transfer will be based on the existing modules in the system. However, going beyond the borders of the own institution would require proper protection of the database and table containing the relation between the deidentification ID and the real patient credentials. Furthermore, the system architecture and security should be considered carefully to ensure the safety of the system in delivering images with embedded patient data. For those reason, communication within our systems was still limited to one hospital only but currently in further development to enable collaborative studies involving many health institutions.
Table 2 Fields in the DICOM header defined to be de-identified

\begin{tabular}{|c|c|c|c|}
\hline Tag ID & Tag name & Tag ID & Tag name \\
\hline 0008,0020 & StudyDate & 0008,1060 & NameOfPhysicianReadingStudy \\
\hline 0008,0021 & SeriesDate & 0008,1062 & PhysicianReadingStudyIDSequence \\
\hline 0008,0022 & AcquisitionDate & 0008,1070 & OperatorsName \\
\hline 0008,0023 & ContentDate & 0010,0010 & PatientsName \\
\hline 0008,0024 & OverlayDate & 0010,0020 & PatientID \\
\hline 0008,0025 & CurveDate & 0010,0021 & IssuerOfPatientID \\
\hline $0008,002 \mathrm{~A}$ & AcquisitionDatetime & 0010,0030 & PatientsBirthDate \\
\hline 0008,0030 & StudyTime & 0010,0032 & PatientsBirthTime \\
\hline 0008,0031 & SeriesTime & 0010,0040 & PatientsSex \\
\hline 0008,0032 & AcquisitionTime & 0010,1000 & OtherPatientIDs \\
\hline 0008,0033 & ContentTime & 0010,1001 & OtherPatientNames \\
\hline 0008,0034 & OverlayTime & 0010,1005 & PatientsBirthName \\
\hline 0008,0035 & CurveTime & 0010,1010 & PatientsAge \\
\hline 0008,0050 & AccessionNumber & 0010,1040 & PatientsAddress \\
\hline 0008,0080 & InstitutionName & 0010,1060 & PatientsMothersBirthName \\
\hline 0008,0081 & InstitutionAddress & 0010,2150 & CountryOfResidence \\
\hline 0008,0090 & ReferringPhysiciansName & 0010,2152 & RegionOfResidence \\
\hline 0008,0092 & ReferringPhysiciansAddress & 0010,2154 & PatientsTelephoneNumbers \\
\hline 0008,0094 & ReferringPhysiciansTelephoneNumber & 0020,0010 & StudyID \\
\hline 0008,0096 & ReferringPhysicianIDSequence & 0038,0300 & CurrentPatientLocation \\
\hline 0008,1040 & InstitutionalDepartmentName & 0038,0400 & PatientsInstitutionResidence \\
\hline 0008,1048 & PhysicianOfRecord & 0040,A120 & DateTime \\
\hline 0008,1049 & PhysicianOfRecordIDSequence & 0040,A121 & Date \\
\hline 0008,1050 & PerformingPhysiciansName & 0040,A122 & Time \\
\hline 0008,1052 & PerformingPhysicianIDSequence & 0040,A123 & PersonName \\
\hline
\end{tabular}


In conclusion, we have devised a vendor neutral system that supports data distribution and de-identification tasks in an easy, robust and user friendly way, providing a fast process in an easy setup environment while still being able to maintain a high level of security and stability. Its ability to run different de-identification processes in parallel pipelines accessible through a simple web interface is a major advantage in both clinical practice and clinical research setting.

Acknowledgments The authors would like to thank Dr. E.J.K. Noach for her editorial help in preparing the manuscript.

Funding This work is part of the ENACT project which is funded by the ZonMw Innovative Medical Devices Initiative (IMDI) call under project registration number 104002003 .

Open Access This article is distributed under the terms of the Creative Commons Attribution License which permits any use, distribution, and reproduction in any medium, provided the original author(s) and the source are credited.

\section{References}

1. Huang, H. K., Enterprise PACS and image distribution. Comput Med Imaging Graph 27:241-253, 2003. doi:10.1016/S0895-6111(02) 00078-2.

2. Rajala, T., Savio, S., Penttinen, J., et al., Development of a research dedicated archival system (TARAS) in a university hospital. $J$ Digit Imaging 24(5):864-873, 2011. doi:10.1007/s10278-010-9350-1.

3. Bandon, D., Lovis, C., Geissbühler, A., and Vallée, J. P., Enterprisewide PACS: Beyond radiology, an architecture to manage all medical images. Acad Radiol 12(8):1000-1009, 2005. doi:10.1016/j.acra. 2005.03.075.

4. van de Wetering, R., Batenburg, R., Oudkerk, M., van Ooijen, P. M. A., Brinkkemper, S., and Scheper, W., A situational alignment framework for PACS. J Digit Imaging 24(6):979-992, 2011. doi:10.1007/ s10278-011-9368-z.

5. Stoian, A., Ivan, R., Stoian, I., Marichescu, A., Current trends in medical imaging acquisition and communication. In: Proceedings of the IEEE international conference on automation, quality and testing, robotics. 2008. Volume 3: 94-99. doi: 10.1109/AQTR.2008.4588890.
6. Achenbach, S., Intranet and radiology: a critical appraisal of radiological applications of Intranet technology. Eur Radiol 12(2):485490, 2002. doi:10.1007/s003300100969.

7. Kotter, E., Baumann, T., Jäger, D., and Langer, M., Technologies for image distribution in hospitals. Eur Radiol 16(6):1270-1279, 2006. doi:10.1007/s00330-006-0153-1.

8. DICOM Standards Committee. The DICOM Standard. National Electrical Manufacturers Association. Available via http://medical. nema.org/standard.html. Accessed October 2013.

9. Langer, S. G., Challenges for data storage in medical imaging research. J Digit Imaging 24(2):203-207, 2011. doi:10.1007/s10278010-9311-8.

10. Langer, S., Issues surrounding PACS archiving to external, thirdparty DICOM archives. J Digit Imaging 22(1):48-52, 2009. doi:10. 1007/s10278-008-9125-0.

11. Susilo, W., Win, K.T. Security and Access of Health Research Data. J. Med. Syst. 31(2). doi: 10.1007/s10916-006-9035-y, 2007.

12. Hsu, C.L., Lee, M.R., Su, C.H. The role of privacy protection in healthcare information systems adoption. J. Med. Syst. 31(2). doi: 10.1007/s10916-013-9966-z, 2013.

13. Chen, C.L., Yang, T.T., Shih, T.F. A secure medical data exchange protocol based on cloud environment. J. Med. Syst. 38(9). doi: 10. 1007/s10916-014-0112-3, 2014.

14. Oracle and Java. Oracle Corporation. Available via http://www. oracle.com/us/technologies/java/overview/index.html. Accessed October 2014.

15. Microsoft SQL Server. Microsoft Corporation. Available via http:// www.microsoft.com/sqlserver/en/us/default.aspx. Accessed October 2014.

16. PHP: Hypertext Preprocessor. The PHP Group. Available via http:// www.php.net. Accessed October 2014.

17. Fernández-Alemán, J.L., Seva-Llor, C. L., Toval, A., Ouhbi, S., Fernández-Luque, L. Free Web-based personal health records: An analysis of functionality. J. Med. Syst. 37(6). DOI: 10.1007/s10916013-9990-z, 2013.

18. CTP-The RSNA Clinical Trial Processor. Radiological Society of North America, Inc. Available via http://mircwiki.rsna.org/index.php?title= CTP-The RSNA Clinical Trial Processor. Accessed October 2014.

19. RSNA.org. Radiological Society of North America, Inc. Available via http://www.rsna.org. Accessed October 2014.

20. Mendelson, D. S., Bak, P. R., Menschik, E., and Siegel, E., Informatics in radiology: Image exchange: IHE and the evolution of image sharing. Radiographics 28(7):1817-1833, 2008. doi:10. 1148/rg.287085174.

21. DICOM Standards Committee, Working Group 18 Clinical Trials (2008) Digital Imaging and Communications in Medicine (DICOM) Supplement 142: Clinical Trial De-Identification Profiles, Final Text, Available via ftp://medical.nema.org/medical/ dicom/Final/sup142 ft.pdf. Accessed October 2014. 